\title{
The Chicken Aorta as a Simulation-Training Model for Microvascular Surgery Training
}

\author{
Savitha Ramachandran ${ }^{1}$, Christopher Hoe-Kong Chui ${ }^{2}$, Bien-Keem Tan ${ }^{2}$ \\ ${ }^{1}$ Department of Plastics and Reconstructive Surgery, KK Women's and Children's Hospital, Singapore; ${ }^{2}$ Department of Plastics and \\ Reconstructive Surgery, Singapore General Hospital, Singapore
}

As a technically demanding skill, microsurgery is taught in the lab, in the form of a course of variable length (depending on the centre). Microsurgical training courses usually use a mixture of non-living and live animal simulation models. In the literature, a plethora of microsurgical training models have been described, ranging from low to high fidelity models. Given the high costs associated with live animal models, cheaper alternatives are coming into vogue. In this paper we describe the use of the chicken aorta as a simple and cost effective low fidelity microsurgical simulation model for training.

Keywords Microsurgery / Education / Animal experimentation / Models, animal

\author{
Correspondence: \\ Savitha Ramachandran \\ Department of Plastics and \\ Reconstructive Surgery, KK Women's \\ and Children's Hospital, 100 Bukit \\ Timah Road, 229899 Singapore \\ Tel: +65-8121-1485 \\ Fax: +65-6394-1154 \\ E-mail: Savitha.rama@gmail.com
}

Received: 15 Feb 2013 • Revised: 18 Apr 2013 • Accepted: 13 May 2013

pISSN: 2234-6163 • elSSN: 2234-6171 • http://dx.doi.org/10.5999/aps.2013.40.4.327 • Arch Plast Surg 2013;40:327-329

\begin{abstract}
This article was presented at the inaugural meeting of the International Microsurgery Simulation Society on June 30, 2012 at the Queen Mary, University of London Mile End Campus, in London, UK.

The authors would like to thank Miss Song, Microsurgical Laboratory Technologist and Dr. Chew Khong Yik for their contributions to this article.

No potential conflict of interest relevant to this article was reported.
\end{abstract}

\section{INTRODUCTION}

Microvascular surgery is a skill that can be challenging and intimidating to the junior trainee. The factors contributing to reduced opportunity to gain clinical practice in microvascular surgery such as high morbidity, an increased litigation rate, and the increasing cost of operating theatre time, have been well documented in the literature $[1,2]$.

In most microsurgical training courses, live anesthetized rats are used [3]. The increasing cost of these rats, in the form of staff and maintenance, has made running of these courses costly [3]. Therefore, cheaper alternatives are always useful. Furthermore, a high fidelity rat model may be more useful for experienced trainees as the vessels in the rat have a much smaller lumen and are much more delicate. For novices, a low fidelity model may be more useful for practicing basic suturing and knot tying in microsurgery.

In this article, we demonstrate the use of the chicken aorta as a low fidelity model for the training of basic microsurgical suturing techniques.

Copyright $\odot 2013$ The Korean Society of Plastic and Reconstructive Surgeons

This is an Open Access article distributed under the terms of the Creative Commons Attribution Non-Commercial License (http://creativecommons.org/

licenses/by-nc/3.0/) which permits unrestricted non-commercial use, distribution, and reproduction in any medium, provided the original work is properly cited.

www.e-aps.org 


\section{Fig. 1. Vessel preparation of the chicken aorta}

(A) Removal of blood clot. (B) Trimming of adventitia.
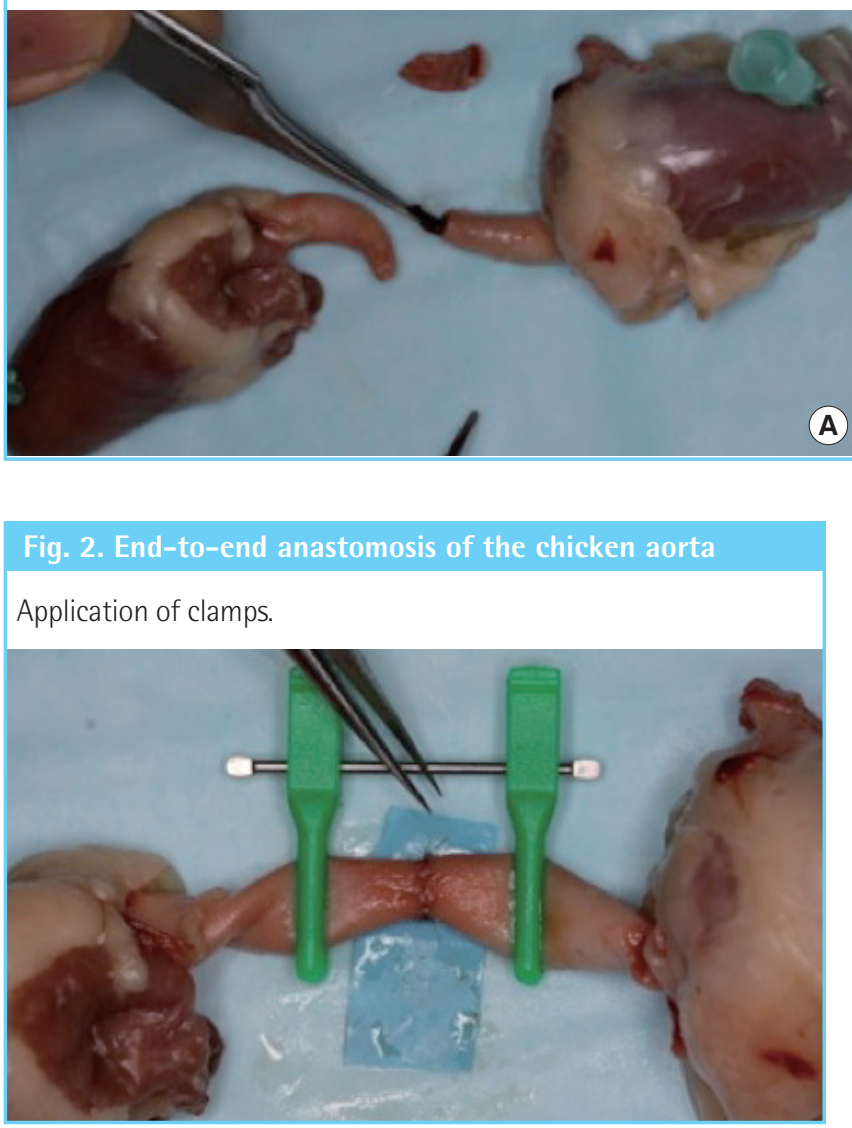

\section{IDEAS AND INNOVATIONS}

The chicken aorta is cheap and easily obtained. In Singapore, as people seldom purchase the heart, it is often available in abundance in the local wet market for only a small fee.

The average size of the lumen of the chicken aorta is $4.5 \mathrm{~mm}$ and the average length of the arch is about $2 \mathrm{~cm}$. Only minimal preparation of the vessels is needed (Fig. 1).

After vessel preparation, the vessels are opposed and held with double clamps (Fig. 2). Suturing with 8.0 ethilon can be done, and both simple interrupted and continuous suturing techniques can be practiced (Fig. 2). Upon completion of the anastomosis, vessel patency can be assessed by injecting methylene blue into the anastomosis (Fig. 3).

\section{DISCUSSION}

The chicken heart is easily obtainable and cheap. In Singapore, the average cost of 1 kilogram of 18 chicken hearts is SGD \$7, which is 40 cents/heart. They are easily available at any wet market. The chicken hearts require minimal preparation upon

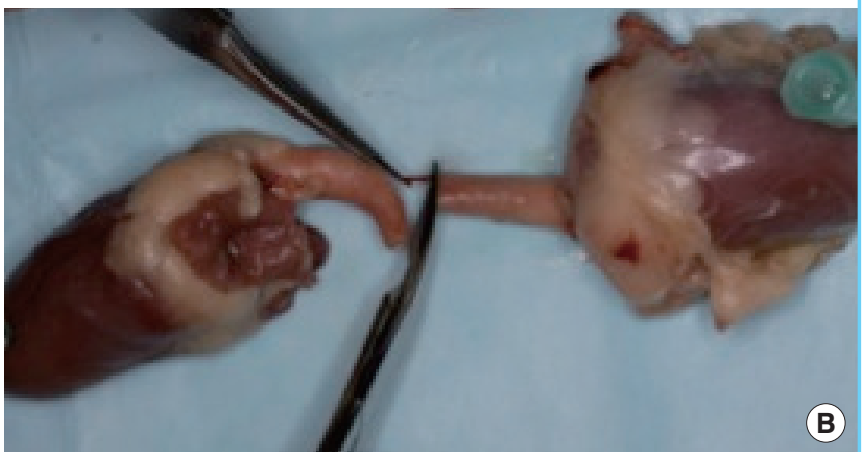

Fig. 3. Patency test with methylene blue

Injection of methylen blue.

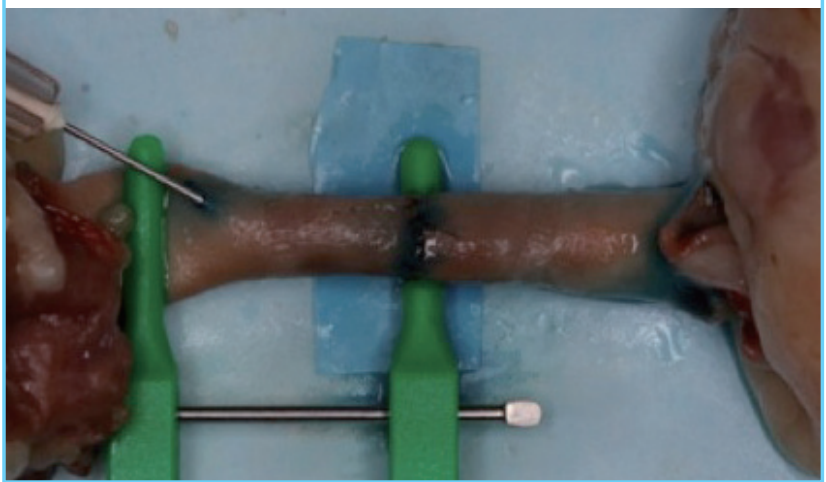

purchase. A variety of suturing techniques can be demonstrated and practiced on the chicken aorta as illustrated by Figs. 1-3.

The slightly larger lumen size $(4 \mathrm{~mm})$ of the chicken aorta makes it more suitable for demonstration and practicing of microsurgical suturing techniques than chicken wing and thigh vessels. The larger lumen size makes it less intimidating for the beginner and will build confidence in the novice microsurgeon. The ability to check vessel patency is also an added advantage of this model (Fig. 3). The use of the chicken aorta also helps to reduce reliance on live animal models, thereby reducing the cost of training. Although there is a risk of transmission of disease from raw chicken, given that chicken is a consumable, handling of the chicken with universal precautions renders very low risk of disease transmission $[4,5]$.

Due to its low cost and easy availability, and low risk as a biohazard, we recommend using the chicken aorta in the early stages of microsurgical training to practice basic suturing skills and to build confidence in the novice trainee.

A randomized controlled study comparing the trainees' feedback on a chicken aorta model to other low fidelity models will be the next step in defining the role of this training model in the 
field of microsurgical education.

\section{REFERENCES}

1. Dean B, Pereira E. Surgeons and training time [Internet]. London: BMJ Careers; 2011 Oct 26 [cited 2013 Jun 18]. Available from: http://careers.bmj.com/careers/advice/ view-article.html?id=20005162.

2. Pereira EA, Dean BJ. British surgeons' experiences of mandatory online workplace-based assessment. J R Soc Med
2009; 102:287-93.

3. Schoffl H, Froschauer SM, Dunst KM, et al. Strategies for the reduction of live animal use in microsurgical training and education. Altern Lab Anim 2008;36:153-60.

4. Ta YT, Nguyen TT, To PB, et al. Prevalence of Salmonella on chicken carcasses from retail markets in Vietnam. J Food Prot 2012;75:1851-4.

5. Goh SG, Kuan CH, Loo YY, et al. Listeria monocytogenes in retailed raw chicken meat in Malaysia. Poult Sci 2012;91: 2686-90. 